\title{
Activity-Dependent Spine Morphogenesis: A Role for the Actin-Capping Protein Eps8
}

\author{
Eleanna Stamatakou, ${ }^{1}$ Aude Marzo, ${ }^{1}$ Alasdair Gibb, ${ }^{2}$ and Patricia C. Salinas ${ }^{1}$ \\ Departments of ${ }^{1}$ Cell and Developmental Biology and ${ }^{2}$ Neuroscience, Physiology and Pharmacology, University College London, London WC1E 6BT, United \\ Kingdom
}

Neuronal activity regulates the formation and morphology of dendritic spines through changes in the actin cytoskeleton. However, the molecular mechanisms that regulate this process remain poorly understood. Here we report that Eps8, an actin-capping protein, is required for spine morphogenesis. In rat hippocampal neurons, gain- and loss-of-function studies demonstrate that Eps8 promotes the formation of dendritic spines but inhibits filopodium formation. Loss of function of Eps8 increases actin polymerization and induces fast actin turnover within dendritic spines, as revealed by free-barbed end and FRAP assays, consistent with a role for Eps8 as an actincapping protein. Interestingly, Eps8 regulates the balance between excitatory synapses on spines and on the dendritic shaft, without affecting the total number of synapses or basal synaptic transmission. Importantly, Eps8 loss of function impairs the structural and functional plasticity of synapses induced by long-term potentiation. These findings demonstrate a novel role for Eps 8 in spine formation and in activity-mediated synaptic plasticity.

\section{Introduction}

The formation and growth of dendritic spines, actin-rich protrusions that mainly receive excitatory inputs, are crucial for the assembly of functional neuronal circuits. Increased spine density correlates with an increased number of excitatory synapses, whereas spine growth is associated with changes in synaptic strength (Bourne and Harris, 2008; Segal, 2010). Dendritic spine morphogenesis is critically dependent on actin dynamics, a process that is modulated by signaling molecules (Tada and Sheng, 2006; Schubert and Dotti, 2007) and neuronal activity (Hotulainen and Hoogenraad, 2010; Bosch and Hayashi, 2011). Indeed, long-term potentiation (LTP) promotes the formation and growth of spines through changes in actin dynamics (Carlisle and Kennedy, 2005; Bramham, 2008; Cingolani and Goda, 2008; Bosch and Hayashi, 2011). Interestingly, changes in the actin cytoskeleton are required for the maintenance of LTP (late LTP), but not for its induction (early LTP) (Krucker et al., 2000; Fukazawa et al., 2003; Ramachandran and Frey, 2009). However, the molecular mechanisms by which neuronal activity regulates spine morphogenesis through changes in the actin cytoskeleton remain poorly understood.

\footnotetext{
Received Feb. 29, 2012; revised Dec. 17, 2012; accepted Dec. 18, 2012

Author contributions: E.S., A.G., and P.C.S. designed research; E.S., A.M., and A.G. performed research; E.S., A.M., A.G., and P.C.S. analyzed data; E.S., A.M., A.G., and P.C.S. wrote the paper.

This work was supported by the Biotechnology and Biological Sciences Research Council, the Medical Research Council, and the Wellcome Trust. We thank Dr. Yukiko Goda for the EGFP-actin construct, Dr. Jeffrey Miller for the Eps8-myc construct, Dr. Riccardo Fodde for the pSuper and the scrambled shRNA vectors, and members of our laboratory for useful discussion and comments on the manuscript.

The authors declare no competing financial interests.

This article is freely available online through the J Neurosci Open Choice option.

Correspondence should be addressed to Dr. Patricia C. Salinas, Department of Cell and Developmental Biology, University College London, Rockefeller Building 5th Floor, 21 University Street, London WC1E 6BT, United Kingdom. E-mail:p.salinas@ucl.ac.uk.

DOI:10.1523/JNEUROSCI.0998-12.2013

Copyright $\odot 2013$ the authors $\quad 0270-6474 / 13 / 332661-10 \$ 15.00 / 0$
}

Actin-capping proteins play a key role in the assembly and elongation of actin filaments. Epidermal growth factor receptor pathway substrate 8 (Eps8) is a multifunctional protein that regulates the actin cytoskeleton (Scita et al., 1999; Disanza et al., 2004; Disanza et al., 2006; Offenhauser et al., 2006). Eps8 directly affects actin dynamics through its barbed end capping and bundling activities (Disanza et al., 2006; Hertzog et al., 2010) or indirectly via Rac1 activation (Scita et al., 1999; Innocenti et al., 2002; Offenhauser et al., 2004). Eps8 can therefore modulate both actin dynamics and organization. These activities are controlled by interactions with different proteins and are highly dependent on the cellular context (Vaggi et al., 2011). Interaction with Abi1/2 promotes its capping activity or its ability to activate Rac1 (Scita et al., 1999; Scita et al., 2001; Innocenti et al., 2002; Disanza et al., 2004; Menna et al., 2009), whereas its interaction with IRSp53 induces actin bundling (Disanza et al., 2006). In neurons, Eps8 is prominently enriched in the axonal growth cone, where it inhibits filopodium formation through its capping activity (Menna et al., 2009; Vaggi et al., 2011). Although Eps8 localizes to both the presynaptic and postsynaptic sides (Offenhauser et al., 2006; Proepper et al., 2007; Sekerkova et al., 2007), its role in synapse formation has not been examined.

Here, we demonstrate that Eps8, which is enriched at dendritic spine heads, is required for spine morphogenesis and activity-mediated synaptic plasticity. Eps8 silencing increases actin polymerization and turnover within dendritic spines, consistent with its actin-capping activity. In addition, we show that Eps8 increases the localization of excitatory synapses on dendritic spines with a concomitant decrease in the number of shaft excitatory synapses. Moreover, Eps8 loss of function impairs the structural and functional changes at synapses induced by chemical LTP (cLTP). In summary, our studies reveal a novel role for the actin-capping protein Eps8 in the localization of excitatory 
synapses and in the plasticity of dendritic spines induced by chemical LTP.

\section{Materials and Methods}

Hippocampal neuronal cell cultures and transfection. Primary neurons were prepared from E18 embryos of Sprague Dawley rats according to Dotti et al. (1988) and were cultured at high density $\left(250\right.$ cells $/ \mathrm{mm}^{2}$ ) in Neurobasal medium supplemented with N2 (Invitrogen), B27 (Invitrogen), D-glucose, and L-glutamine. Cells were transfected either with calcium phosphate (Dudek et al., 2001) or with Lipofectamine 2000 (Invitrogen) at 7-8 DIV and analyzed at 13-14 DIV. To examine endogenous Eps8 localization on spines (Fig. 1B), low-density cultures $\left(100\right.$ cells $\left./ \mathrm{mm}^{2}\right)$ transfected using the Amaxa nucleofector kit (Lonza) were used. Constructs used were for EGFP (Clontech), EGFP-actin, Eps8-myc, Eps8D533-821-myc, Eps8TM-myc, scrambled shRNA, and shRNAs against Eps8. Our cotransfection protocols with EGFP-actin were standardized to achieve the maximum cotransfection efficiency (almost $100 \%$ ) using a ratio of $1: 3$.

Immunofluorescence, image acquisition, and analysis. Cultures were fixed with 4\% PFA/4\% sucrose in PBS for $20 \mathrm{~min}$ at room temperature or with $100 \%$ ice-cold methanol for $5 \mathrm{~min}$ at $-20^{\circ} \mathrm{C}$. Primary antibodies against Eps8 (BD Transduction Laboratories), GFP (Millipore), vGlut1 (Millipore), PSD95 (Thermo Scientific), GluN1 (Synaptic Systems), and surface GluA1 (Calbiochem) were used. For surface GluA1, live cultures were incubated with an antibody against the extracellular domain of GluA1 for $10 \mathrm{~min}$ at $37^{\circ} \mathrm{C}$ before fixation. Secondary antibodies conjugated with Alexa-488, Alexa-568 or Alexa-647 were from Invitrogen. Fluorescence images were captured with an Olympus FV1000 inverted confocal microscope using a $60 \times$ oil-immersion objective $(\mathrm{NA}=1.40)$, producing image stacks $162.7 \times$ $162.7 \mu \mathrm{m}$ with an average $z$ depth of $\sim 4 \mu \mathrm{m}$. A total of $15-25$ cells per condition were acquired and analyzed. Images were analyzed using Volocity software (Improvision). For each cell, 3 or 4 dendrites were analyzed from maximum projection images. Filopodium number, spine number, and size were measured manually. Filopodia were defined as thin protrusions without a distinguishable head, thin spines as thin protrusions with a distinguishable head,

stubby spines as short protrusions without a neck, and mushroom spines as protrusions with a short neck and a distinguishable head. Spines were classified as irregular when their spine head did not have a typical mushroom shape. Synapses were defined by the apposition of presynaptic and postsynaptic markers, such as vGlut1 and GluN1.

Fluorescent recovery after photobleaching. Fluorescent recovery after photobleaching (FRAP) experiments were performed according to Koskinen et al. (2012) with minor modifications. Image sequences were captured using an Olympus FV1000 inverted confocal microscope with a $60 \times$ oil-immersion objective $(\mathrm{NA}=1.40)$. FRAP assays were performed at $37^{\circ} \mathrm{C}$ and $5 \% \mathrm{CO}_{2}$ in culture medium. Images were captured using maximum $10 \%$ of laser power $(488 \mathrm{~nm})$ in a $256 \times 256$ format, $600 \mathrm{~Hz}$ speed, 2-line averaging, 2.0 arbitrary unit pinhole and 3.0 optical zoom. To specify the area of photobleaching, we traced a squared ROI with a maximum area of $7 \mu \mathrm{m}^{2}$, big enough to cover a single spine. The FRAP protocol was as follows: before bleaching 3 frames with $2 \mathrm{~s}$ interval were ${ }^{* *} p<0.001$. ns, Not significant.
B

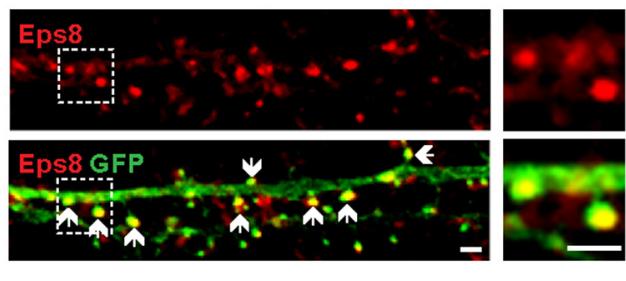

D

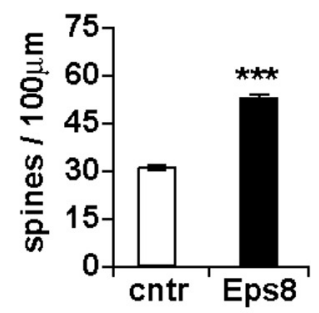

$E$

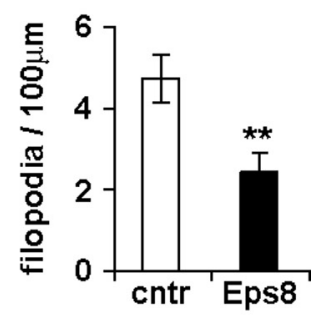

H

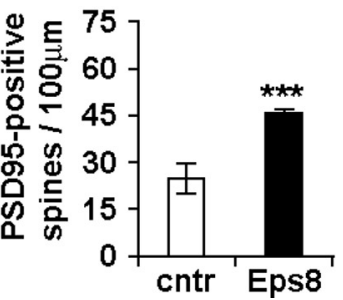

Figure 1. Eps8 is localized at synapses and promotes spine formation. $\boldsymbol{A}$, Eps 8 is enriched in synaptosomes isolated from adult mouse brains and specifically in the PSD fraction. Brain, Brain homogenate; Syn, synaptosomes; SMF, synaptosomal membrane fraction (presynaptic and soluble postsynaptic fraction). $\boldsymbol{B}$, Endogenous Eps8 localizes into dendritic spines (arrows). Scale bars, 2 increases the number of spines $(\boldsymbol{D})$, whereas it decreases filopodium density $(\boldsymbol{E})$, and spine size is unaffected $(\boldsymbol{F})$. Eps8 specifically increases the number of stubby spines (arrowheads; $\boldsymbol{G}$ ) and the number of spines containing PSD95 (asterisks; $\boldsymbol{H}$ ). ${ }^{* *} p<0.01$,

captured followed by photobleaching using 200 pulses with $100 \%$ laser power (maximum bleaching time $6 \mathrm{~s}$ ). Subsequently, 20 frames with $2 \mathrm{~s}$ intervals were captured to detect rapid recovery of fluorescence. This was followed by the acquisition of another 20 frames with $5 \mathrm{~s}$ intervals to avoid laser overexposure of the cells and 10 more frames with $20 \mathrm{~s}$ intervals until full recovery of fluorescence was obtained. The total fluorescence intensity of the ROI for each time point was measured using Volocity software (Improvision). The average fluorescence intensity of the first three frames (before photobleaching) was used to normalize the intensity values for each time point. The recovery $t_{1 / 2}$ was determined from the average scatter plot, and the first-order rate constant $\left(k_{\text {obs }}\right)$ was calculated using the equation: $k_{\text {obs }}=0.693 / t_{1 / 2}$ (Koskinen et al., 2012).

Free-barbed end assay. Free-barbed end assays were performed as previously described (Gu et al., 2010; Marsick and Letourneau, 2011) with minor modifications. In brief, neurons were exposed to purified nonmuscle rhodamine-conjugated G-actin (cytoskeleton) that was diluted at 
$0.45 \mu \mathrm{M}$ final concentration in saponin permeabilization buffer $(20 \mathrm{~mm}$ HEPES, $138 \mathrm{~mm} \mathrm{KCl}, 3 \mathrm{~mm}$ EGTA, $4 \mathrm{~mm} \mathrm{MgCl}_{2}, 1 \%$ BSA, $0.2 \mathrm{mg} / \mathrm{ml}$ saponin, $0.5 \mathrm{~mm}$ DTT, and $1 \mathrm{~mm} \mathrm{ATP,} \mathrm{pH} \mathrm{7.5)} \mathrm{for} 2 \mathrm{~min}$. Cultures were immediately fixed with 4\% PFA in PHEM buffer (60 mM PIPES, $25 \mathrm{~mm}$ HEPES, 10 mM EGTA, 2 mM $\mathrm{MgCl}_{2}, 0.12 \mathrm{M}$ sucrose, $\mathrm{pH}$ 7.0), treated with $0.1 \mathrm{M}$ glycine in PBS for $10 \mathrm{~min}$ and then stained for GFP (Millipore) and F-actin using phalloidin conjugated with Alexa-647 (Invitrogen).

Eps 8 truncated construct. Eps $8 \Delta 533-821$ was created by PCR using the Eps8-myc construct as template. Primers used were as follows: forward, 5'-AAGTACATCGATATGAATGGTCATATGTCTAACC GC-3'; and reverse, 5'-AAGTACCTCGAGTCATAGGTCTTCGGAGATTAGCT TTTGCTCG-3'. The PCR product was then inserted onto PCS2+ vector.

A mutant Eps8 carrying three point mutations (V729A, T731A, and W732A) called Eps8TM was created using the QuikChangeLightning Site-Directed Mutagenesis kit (Stratagene) following the manufacturer's guidelines. Primers used were as follows: forward, $5^{\prime}$-TCCTCACCGGAA GAAGCAAAGGCTGCGCTGCAGTCAAAGGGA-3'; and reverse, 5'-TCCCTTTGACTGCAGCGCAGCCTTTGCTTCTTCCGGTGAGGA-3'.

shRNA constructs for Eps8. Each shRNA against rat Eps8, containing a $21 \mathrm{nt}$ target sequence was cloned into the pSuper vector (Oligo Engine). Target sequences were for shRNA\# $1: 5^{\prime}$-AGGCCCTTTATGAACAAAGT T-3'; for shRNA\#2: 5'-ACATGGATTCAACCTTCTGTT-3'; and for shRNA\#3: 5'-GAAATACGCCAAATCCAAGTT-3'. A combination of the three shRNAs was used to achieve a significant level of knockdown, as assessed by the total levels of endogenous Eps8 in somas normalized to soma volume and the number of endogenous Eps8 puncta per dendritic length in hippocampal neurons.

Synaptosomal preparation. Synaptosomes were prepared from adult mouse brains of either sex using a sucrose gradient protocol, as previously described (Sahores et al., 2010). Equal amounts of proteins were run onto a SDS-PAGE. Primary antibodies against Eps8 (BD Transduction Laboratories), Syntaxin1 (Sigma), and PSD95 (Thermo Scientific) were used. Band intensity was quantified using ImageJ software (National Institutes of Health).

cLTP. LTP was induced in 13-14 DIV hippocampal cultures using a cLTP protocol (200 $\mu \mathrm{M}$ glycine for $10 \mathrm{~min}$ in the absence of $\mathrm{Mg}^{2+}$ ) (Oh and Derkach, 2005; Fortin et al., 2010). After cLTP induction, cultures were returned to control solution for $1 \mathrm{~h}$ before fixation or for $40 \mathrm{~min}$ before AMPA receptor-mediated miniature (mEPSC) recordings. Briefly, before cLTP induction, cultures were incubated for $20 \mathrm{~min}$ in control solution ( $125 \mathrm{~mm} \mathrm{NaCl}, 2.5 \mathrm{~mm} \mathrm{KCl}, 2 \mathrm{mM} \mathrm{CaCl}_{2}, 1 \mathrm{~mm} \mathrm{MgCl}_{2} 5$ mM HEPES, 33 mm glucose, $0.5 \mu \mathrm{M}$ TTX, $20 \mu \mathrm{m}$ AP5, $20 \mu \mathrm{m}$ bicuculline, and $3 \mu \mathrm{M}$ strychnine, $\mathrm{pH}$ 7.4). cLTP was then induced by treating the cultures for $10 \mathrm{~min}$ with glycine in the absence of $\mathrm{Mg}^{2+}$, TTX, and AP5 (125 mm NaCl, $2.5 \mathrm{~mm} \mathrm{KCl,} 5 \mathrm{~mm} \mathrm{CaCl}$, 5 mм HEPES, 33 mм glucose, $200 \mu \mathrm{M}$ glycine, $20 \mu \mathrm{M}$ bicuculline, and $3 \mu \mathrm{M}$ strychnine, $\mathrm{pH}$ 7.4) before returning them back to control solution.

Electrophysiology. AMPA receptor-mediated mEPSCs were recorded, using whole-cell patch-clamp configuration, in the presence of $100 \mathrm{nM}$ TTX, $10 \mu \mathrm{M}$ bicuculline, and $50 \mu \mathrm{M}$ AP5, as previously described (Ciani et al., 2011). Approximately equal numbers of cells was recorded from scrambled or Eps8 shRNAs-expressing cells on each day from 12 to 14 DIV cultures.

For recordings for chemical LTP experiments: Krebs extracellular solution was used supplemented with $1 \mathrm{mM} \mathrm{MgCl}_{2}, 0.5 \mu \mathrm{M}$ TTX, $20 \mu \mathrm{M}$ AP5, $20 \mu \mathrm{m}$ bicuculline, and $3 \mu \mathrm{M}$ strychnine. Analyses were performed using a combination of WinEDR and WinWCP software (freely available at http://spider.science.strath.ac.uk/sipbs/software_ses.htm).

Statistical analysis. Values given are mean \pm SE. Data presented correspond to at least three independent experiments. For datasets with normal distribution, ANOVA test was used. For datasets with nonnormal distribution, the Kruskal-Wallis test was used.

\section{Results}

Eps8 is enriched in the postsynaptic compartment and localizes into dendritic spines

To determine the potential role of Eps8 in synapse formation, we first examined its localization at central synapses. We found that
Eps8 is present in both presynaptosomal and postsynaptosomal fractions (Fig. 1A), as previously reported (Offenhauser et al., 2006; Proepper et al., 2007). However, Eps8 is primarily enriched in the postsynaptic density (PSD) compared with the presynaptic fraction (synaptosomal membrane fraction) (Fig. $1 A$ ). To analyze in more detail the localization of endogenous Eps8 within the dendritic compartment, we used hippocampal neurons expressing EGFP-actin, which allows the easy identification of dendritic spines. Consistent with the biochemical results, we observed that endogenous Eps8 is prominent in dendritic spines and is particularly enriched at spine heads (Fig. $1 B$ ). Together, these results demonstrate that Eps8 is enriched at dendritic spines, postsynaptic structures that mainly receive excitatory inputs.

\section{Eps8 promotes the formation of dendritic spines}

The localization of Eps8 on dendritic spines prompted us to examine its potential role in dendritic spine morphogenesis. Gainof-function studies in hippocampal cultures, where Eps8 was expressed together with EGFP-actin (Fig. 1C), reveal that Eps8 induces a $63 \%$ increase in spine density (Fig. $1 D$ ) with a concomitant decrease in filopodia density (Fig. 1E). Although Eps8 does not affect spine size (Fig. $1 F$ ), it does change the morphology of spines, as the number of stubby spines is significantly increased without affecting the number of thin and mushroom spines (Fig. $1 G)$. Similar results were obtained in neurons expressing Eps8 and EGFP (data not shown); we therefore continued our studies using EGFP-actin, which labels more efficiently dendritic spines. In addition, we found that Eps8 increases the number of spines containing PSD95 (Fig. $1 H$ ), a postsynaptic marker that accumulates within spine head during spine maturation (Han and Kim, 2008; McMahon and Diaz, 2011). These results demonstrate that Eps8 induces the formation and maturation of dendritic spines.

\section{Eps8 knockdown decreases spine formation and affects spine morphology}

To further investigate the role of Eps8 in spine morphogenesis, we examined the consequence of Eps8 loss of function by performing shRNA-mediated knockdown (KD) experiments. We used a combination of three shRNAs to obtain a significant level of knockdown, as measured by the level of endogenous Eps8 protein in the cell soma and dendritic processes of shRNAexpressing cells (Fig. 2A). We observed a $79 \%$ reduction in the total intensity level of Eps8 in the cell soma (Fig. 2B) and a 69\% decrease in the Eps8 puncta density on dendrites (Fig. 2C) in neurons expressing Eps8 shRNAs compared with neurons expressing the scrambled shRNA. Thus, Eps8 shRNAs effectively downregulate the levels of endogenous Eps8 protein in cultured hippocampal neurons.

We next examined the impact of Eps8 loss of function on spine morphogenesis (Fig. 2D). Expression of Eps8 shRNAs decreases spine density by $39 \%$ (Fig. $2 E$ ), whereas significantly increases filopodia density by $46 \%$ (Fig. $2 F$ ). In addition, Eps 8 shRNAs-expressing cells have fewer small spines with a concomitant increase in the number of large spines (Fig. 2G), resulting in a significant increase in the average spine size (Fig. $2 \mathrm{H}$ ). We confirmed the specificity of the Eps $8 \mathrm{KD}$, as we found that the three different shRNAs independently decrease spine density (scrambled, $32 \pm 1.63$; shRNA\#1, $21 \pm 1.61$; shRNA\#2, $19 \pm$ 1.59; shRNA\#3, $21 \pm 0.92$ spines per $100 \mu \mathrm{m}$ dendritic length) while increasing spine size (scrambled, $0.66 \pm 0.03 \mu \mathrm{m}$; shRNA\#1, $0.77 \pm 0.02 \mu \mathrm{m}$; shRNA\#2, $0.75 \pm 0.02 \mu \mathrm{m}$; shRNA\#3, $0.74 \pm 0.02 \mu \mathrm{m}$ spine head width). In addition, Eps8 $\mathrm{KD}$ induces an increase in the percentage of spines with filopodia 
growing from spine heads and with irregular spine head shape (Fig. $2 I, J$ ). These results demonstrate that Eps8 is required for spine formation and regulates spine morphology.

Eps8 regulates actin polymerization and turnover within dendritic spines To begin to understand the mechanism by which Eps8 regulates spine morphogenesis, we first examined the effect of a truncated form of Eps8 $(\Delta 533-821)$ that lacks the $\mathrm{SH} 3$ and the actin-binding domain (effector domain) (Fig. 3A). The SH3 domain is required for interaction with Abil (Scita et al., 1999), whereas the effector domain binds directly to actin filaments and induces actin capping and bundling (Hertzog et al., 2010). In contrast to the full-length Eps8, Eps8 $\Delta 533-821$ does not affect spine formation (Fig. $3 B, C$ ). These results demonstrate that Eps8 promotes spine formation through its $\mathrm{C}$ terminus domain containing the actin-binding domain, suggesting that Eps8 modulates spine morphogenesis through changes in the actin cytoskeleton.

To determine whether the capping activity of Eps8 mediates spine formation, we generated a mutant Eps8 (Eps8TM) carrying three single point mutations in the actinbinding domain (V729A, T731A, and W732A). This triple mutant retains the actin bundling activity but is defective in actin capping (Menna et al., 2009). We found that Eps8TM decreases spine density by $42 \%$ whereas increases spine growth by $21 \%$ (Fig. $3 B, C)$, similarly to the phenotype conferred by Eps8 KD (Fig. 2E,H). These results demonstrate that the capping activity of Eps8 is required for spine formation.

To further elucidate the function of Eps8 in spine formation, we examined whether loss of function of Eps8 affects actin dynamics within dendritic spines. To determine the sites of actin polymerization, we performed free-barbed end assays in neurons expressing Eps8 shRNAs (Fig. 3E). The free (uncapped) actin barbed ends were visualized by the incorporation of purified $\mathrm{G}$-actin into actin filaments. We found that in control scrambled shRNA-expressing neurons almost $20 \%$ of the spines have freebarbed ends (Fig. $3 F$ ), as previously reported ( $\mathrm{Gu}$ et al., 2010). In contrast, Eps8 KD leads to a significant increase in the percentage of spines that incorporated fluorescent G-actin (Fig. 3F), thus reflecting an increase in the number of uncapped barbed ends in spines.

To further study the role of Eps8 on actin dynamics, we performed FRAP assays (Fig. 3G) to monitor the turnover of
A
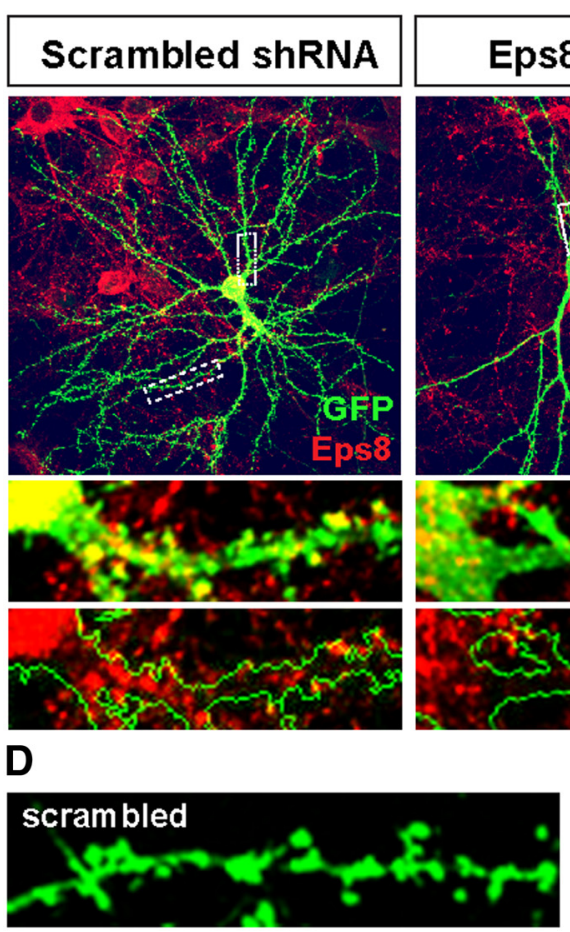

$\mathbf{F}$

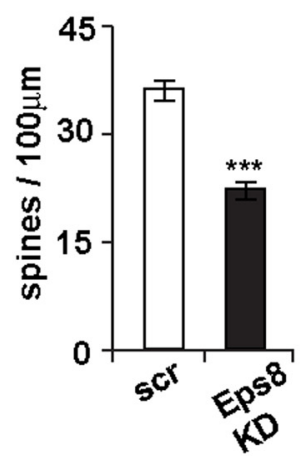

H

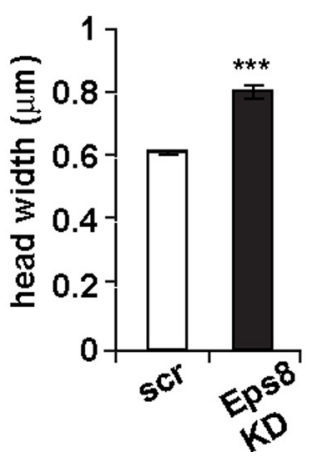

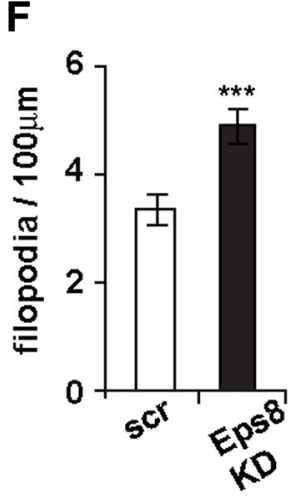

I

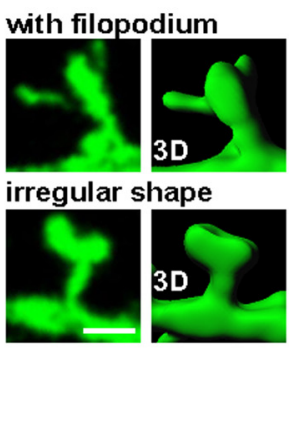

B
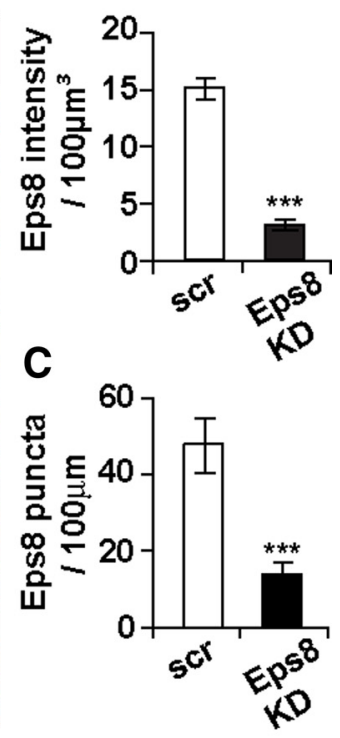

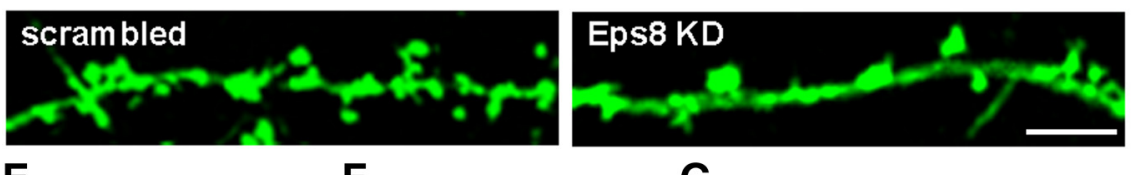

G

Figure 2. Loss of function of Eps8 decreases the formation of dendritic spines. $\boldsymbol{A}$-C , shRNA-mediated knockdown of Eps8 significantly decreases the total intensity level of endogenous Eps8 normalized to soma volume and Eps8 puncta density on dendrites. Scale bars, $10 \mu \mathrm{m}$. D, Eps8 loss of function decreases spine number $(\boldsymbol{E})$ and increases filopodium density $(\boldsymbol{F})$. Scale bar, $5 \mu \mathrm{m} . \mathbf{G}$, Distribution of spines according to their size reveals that loss of function of Eps8 decreases the density of small- and medium-sized spines with a concomitant increase in the density of large spines, thus increasing the average spine size $(\boldsymbol{H})$. Spine head width: small, $\leq 0.7 \mu \mathrm{m}$; medium, $>0.7 \mu \mathrm{m}$ and $\leq 1.0 \mu \mathrm{m}$; large, $>1.0 \mu \mathrm{m}$. The percentage of spines with filopodial protrusions on their head or with abnormal head shape ( $\boldsymbol{I}$, left: fluorescence; right: $3 D$ reconstruction) is increased $(\boldsymbol{J})$. Scale bar, 2 $\mu \mathrm{m} .{ }^{* *} p<0.01$. ${ }^{* *} p<0.001$. 
A

\begin{tabular}{|c|c|c|c|}
\hline Eps8 & PTB & $\mathrm{SH} 3$ & Eff. domain \\
\hline Eps8 $\triangle 4533-821$ & PTB & 12 & PAP \\
\hline Eps8TM & PTB & SH3 & Eff. domain \\
\hline & & כ & \begin{tabular}{c|c}
$\sqrt{729 A}$ & W/32A \\
$731 A$
\end{tabular} \\
\hline
\end{tabular}
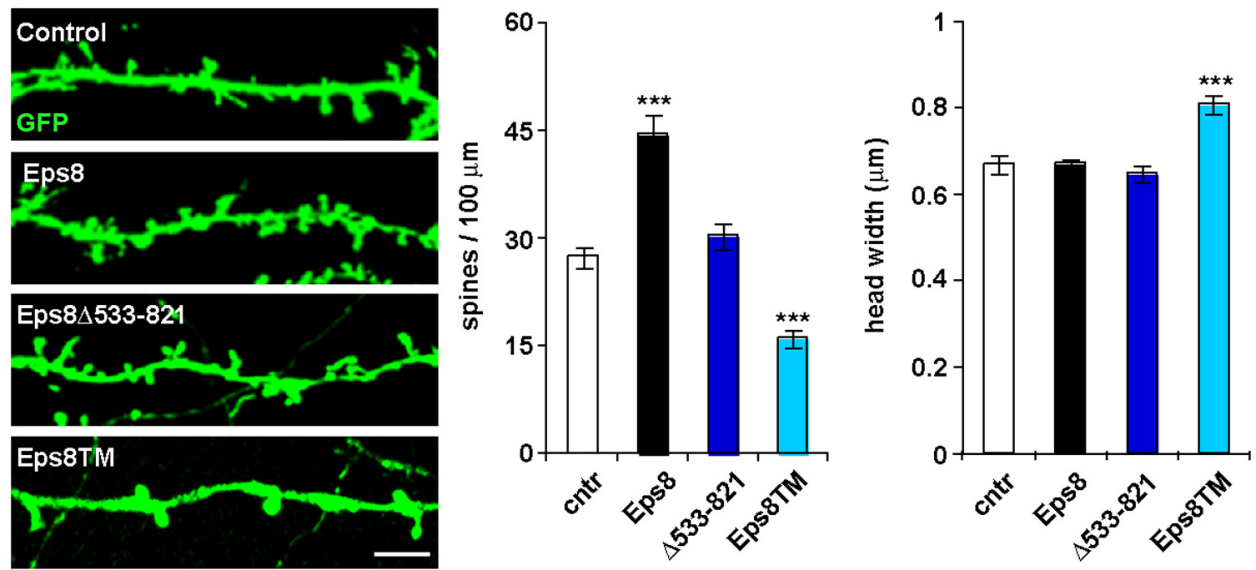

E
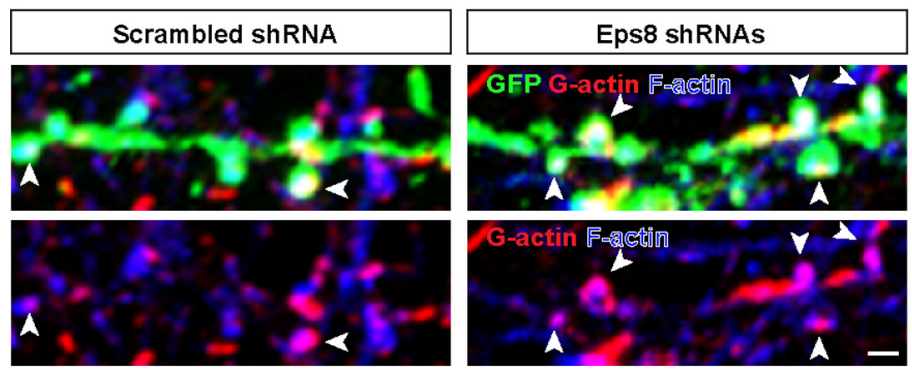

$\mathbf{F}$
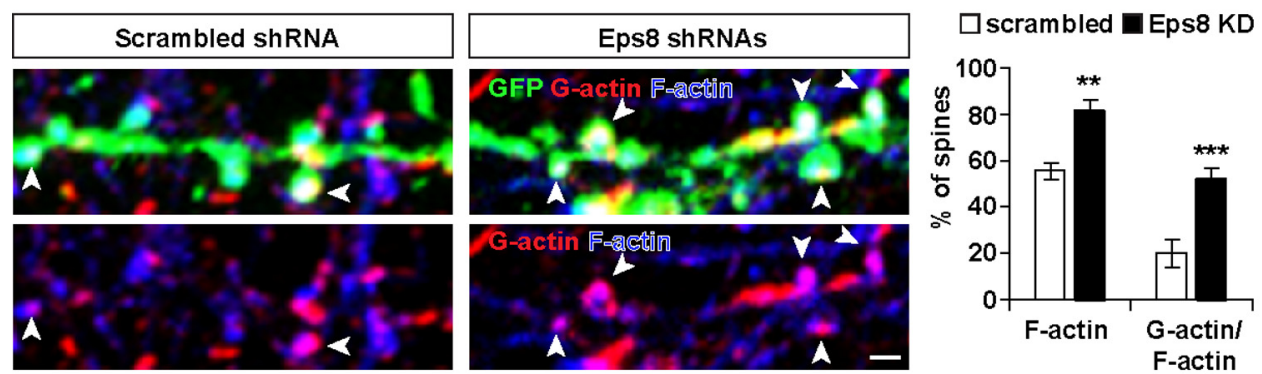

G
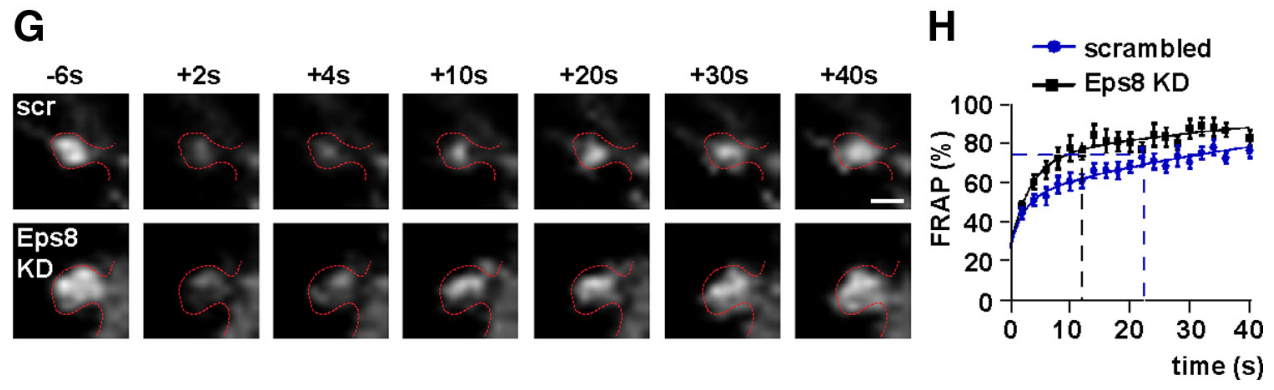

Figure 3. The capping activity of Eps 8 is required for spine formation and its loss of function induces actin polymerization and fast actin turnover. $A$, Illustration of the Eps 8 domains and the constructs used in $\boldsymbol{B}$. Hippocampal neurons expressing Eps8 $\Delta 533-821(\boldsymbol{B})$ do not exhibit changes in spine density $(\boldsymbol{C})$ or spine growth (D). In contrast, expression of a triple mutant (Eps8TM), which lacks a capping activity, decreases spine density $(\boldsymbol{C})$ and increases spine growth $(\boldsymbol{D})$. Scale bar, $5 \mu \mathrm{m}$. Free actin barbed ends were visualized with rhodamineconjugated $\mathrm{G}$-actin. F-actin was labeled with phalloidin $(\boldsymbol{E})$. Scale bar, $1 \mu \mathrm{m}$. Analysis of the sites of active actin polymerization within dendritic spines revealed that Eps $8 \mathrm{KD}$ leads to an increase in the percentage of spines that have $F$-actin and free-barbed ends $(\boldsymbol{F})$. G, FRAP assays were performed by recording spines expressing EGFP-actin from control scramble shRNA and shRNA Eps8 expressing neurons. EGFP-actin was bleached on single spines and fluorescence recovery was followed by time-lapse microscopy. The frames before $(-6 \mathrm{~s})$ and after

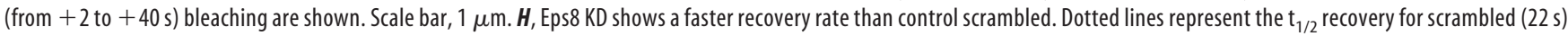
and Eps8 KD (12 s). In spines of scrambled-expressing neurons, the fluorescence recovery of EGFP-actin was complete by $110 \mathrm{~s}$, whereas in Eps 8 shRNAs-expressing neurons complete recovery was achieved within 80 s. ${ }^{*} p<0.05 .{ }^{* *} p<0.01 .{ }^{* *} p<0.001$.

actin filaments at single spines. This assay is based on the fluorescent recovery of EGFP-actin after photobleaching using timelapse imaging (Star et al., 2002; Koskinen et al., 2012). Spines from scrambled shRNA-expressing neurons have a half-time fluorescence recovery $\left(\mathrm{t}_{1 / 2}\right)$ of $22 \mathrm{~s}$, similar to those obtained in previous studies (Star et al., 2002; Koskinen et al., 2012). In contrast, spines from Eps8 KD neurons exhibit a faster EGFP-actin fluorescence recovery with $t_{1 / 2}$ equal to $12 \mathrm{~s}(\mathrm{Fig} .3 \mathrm{H}$ ). Thus, the first-order rate constant for the recovery curve of the control scrambled-expressing cells is $0.031 \mathrm{~s}^{-1}$, whereas for the Eps8 shRNAs-expressing cells is $0.058 \mathrm{~s}^{-1}$. Together, our free-barbed end and FRAP assays demonstrate that Eps8 silencing increases actin polymerization and fast actin turnover, suggesting that Eps8 acts as a capping protein in dendritic spines. 
Eps8 regulates the balance between spine and shaft excitatory synapses To gain more insight into the role of Eps 8 in synapse formation, we examined whether Eps8 affects synapse density. Excitatory synapses were defined by the apposition of the presynaptic marker vGlut1 to the postsynaptic NMDA receptor subunit GluN1 (Fig. $4 A)$. We found that Eps8 gain of function does not affect the total density of excitatory synapses (synapses on spines and on dendritic shafts) (Fig. 4B). Given that Eps8 increases spine density (Fig. 1), these results could suggest that Eps8 augments the proportion of excitatory synapses on spines. Indeed, we found that Eps8 increases the number of synapses on spines, whereas it decreases the number of shaft synapses (Fig. $4 C, D)$. These results demonstrate that Eps8 increases the number of innervated spines at the expense of shaft synapses.

We next investigated the effect of Eps8 $\mathrm{KD}$ on excitatory synapse formation. We found that Eps8 KD does not affect the total density of excitatory synapses (Fig. $4 F)$, similar to the gain of function of Eps8. However, Eps8 KD induces a decrease in the number of synapses on spines with a concomitant increase in the number of shaft excitatory synapses (Fig. $4 G, H)$. Therefore, Eps8 KD decreases the number of dendritic spines, but the total number of excitatory synapses remains unchanged because of a shift toward shaft excitatory synapse formation. These gainand loss-of-function studies demonstrate that Eps8 is required for the proper localization of excitatory synapses on dendritic spines.

Eps8 KD increases the size of dendritic spines. As spine size can be influenced by the content of AMPA receptors (Matsuzaki et al., 2001), we examined the effect of Eps8 KD on the localization of the surface GluA1 AMPAR subunit (sGluA1) at synapses. Synapses were identified by the apposition of vGlut1 to sGluA1 (Fig. 5A). We found that Eps8 loss of function does not affect the total number of synapses containing sGluAl along the dendrites (Fig. 5B). Interestingly, Eps8 silencing increases the number of sGluA1-labeled synapses on the shaft with the concomitant decrease in the number of sGluA1-labeled synapses on spines (Fig. $5 C, D)$. Thus, Eps8 induces a shift in the localization of sGluA1-contaning synapses from spines to the dendritic shaft.

To investigate the functional consequences of Eps8 loss of function at synapses, we performed whole-cell patch-clamp recordings and measured AMPA receptor-mediated mEPSCs in neurons expressing control scrambled and Eps8 shRNAs (Fig. $5 E)$. We found that Eps8 KD does not affect the frequency or the amplitude of mEPSCs (Fig. $5 F, G$ ). These results are consistent

A

E

$\mathbf{F}$
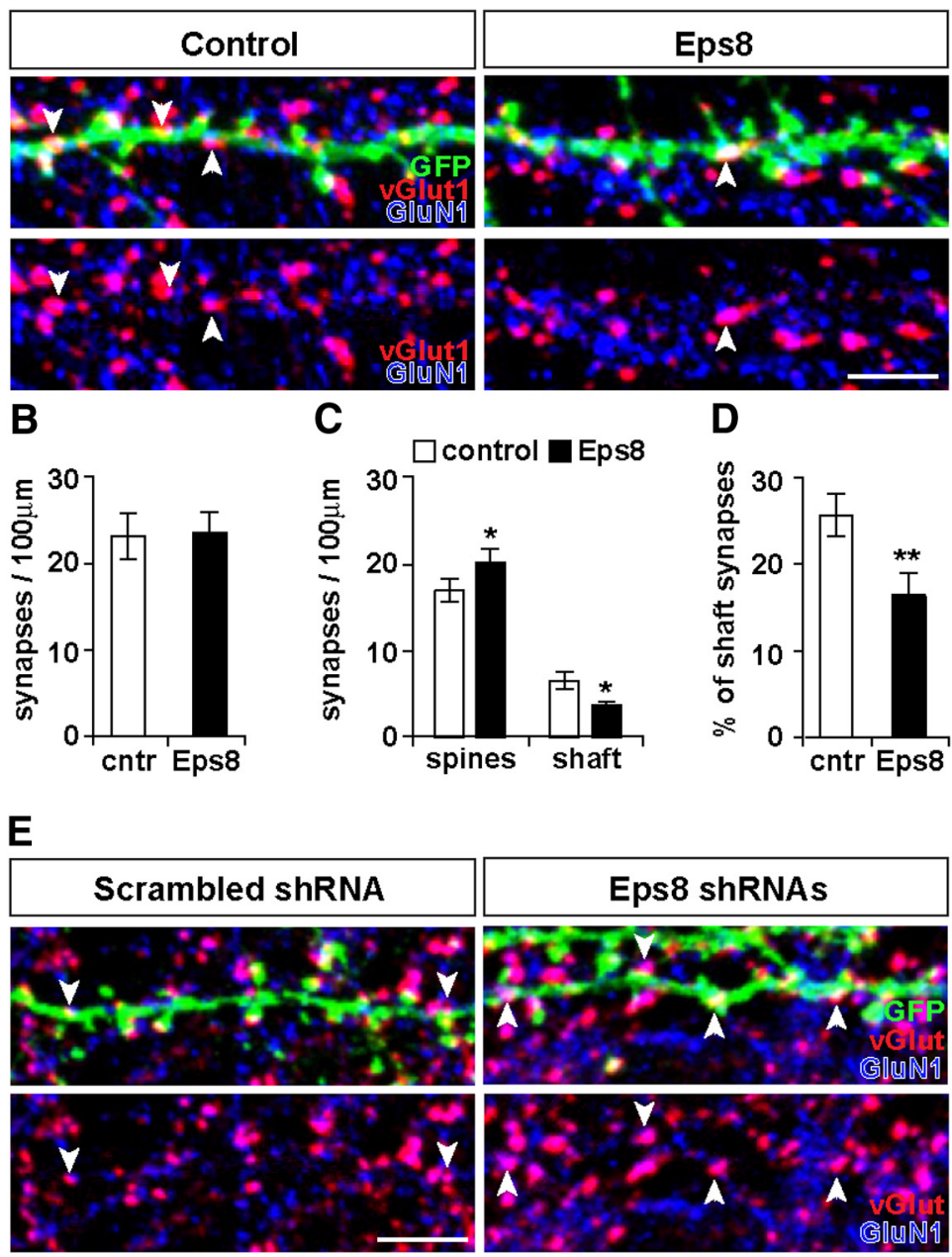

G

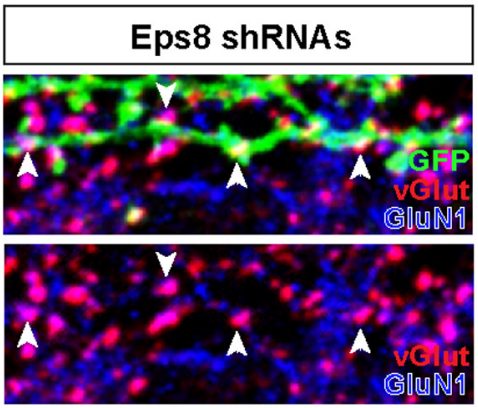

H

$\square$ scrambled $\square$ Eps8 KD
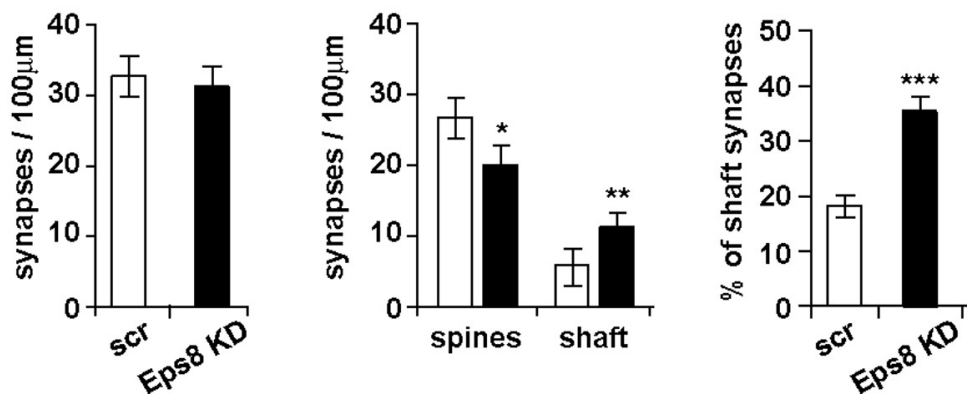

Figure 4. Eps8 regulates the balance between excitatory synapses on spines and on the dendritic shaft. Analysis of synapse density (apposition of vGlut1 to GluN1 puncta) $(\boldsymbol{A})$ reveals that Eps8 expression does not affect total synapse density $(\boldsymbol{B})$ but increases the number of synapses on spines and decreases the number of shaft synapses (A, arrowheads; $\boldsymbol{C}, \boldsymbol{D})$. Scale bar, $5 \mu \mathrm{m}$. $\boldsymbol{E}$, $\boldsymbol{F}$, Eps8 KD does not affect the total number of synapses, but it does decrease the number of synapses on spines $(\boldsymbol{G})$ and increases the number of shaft synapses ( $\boldsymbol{E}$, arrowheads) containing $\operatorname{GluN1}(\boldsymbol{E}, \boldsymbol{G}, \boldsymbol{H})$. Scale bar, $5 \mu \mathrm{m} .{ }^{*} p<0.05$. ${ }^{* *} p<0.01 .{ }^{* *} p<0.001$.

with our findings that Eps8 is required for the localization of excitatory synapses on dendritic spines, but it does not affect the total number GluA1-labeled synapses, therefore not affecting basal synaptic transmission on the neuron.

Eps8 is required for LTP-dependent excitatory synapse formation

Neuronal activity plays a crucial role in the formation and modulation of neuronal circuits. Several studies have demonstrated 
A
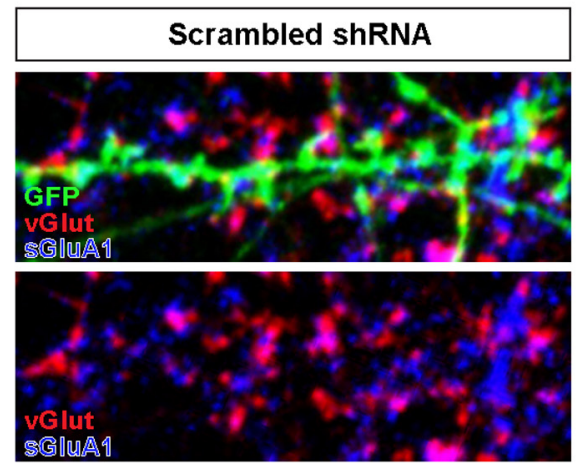

B

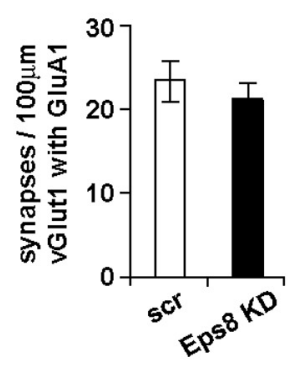

E

\section{Scrambled shRNA}

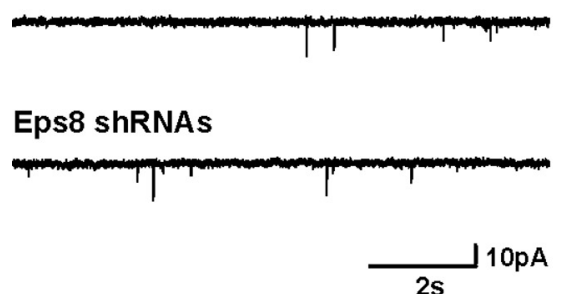

2s

C
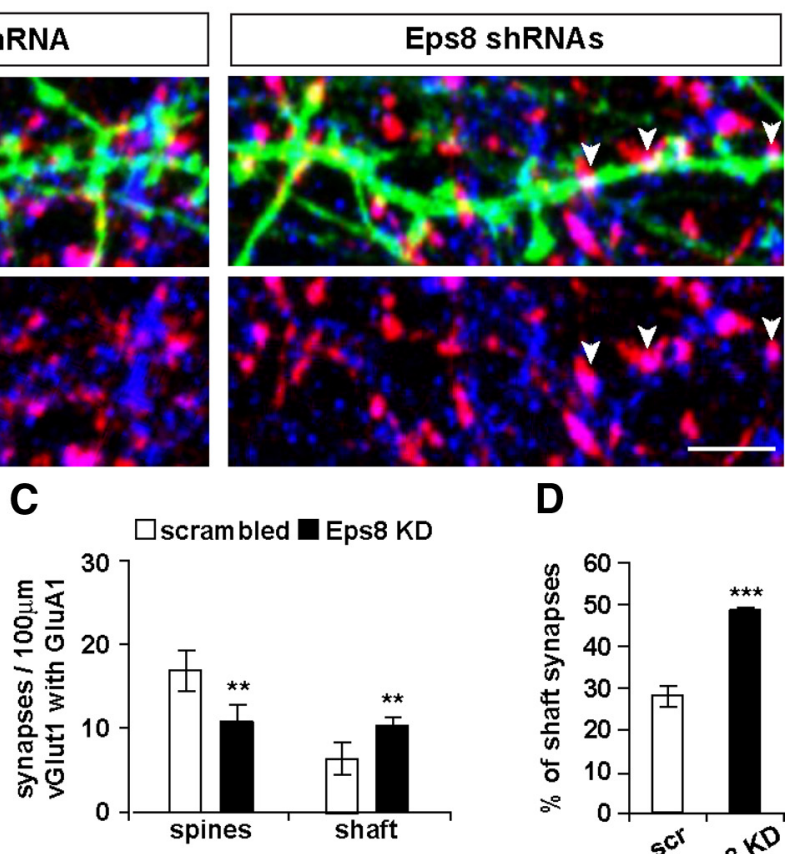

$\mathbf{F}$

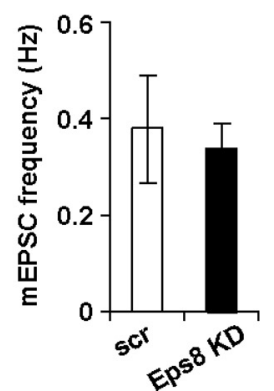

Figure 5. Loss of function of Eps8 does not affect the density of AMPA receptor at excitatory synapses or basal synaptic transmission. $\boldsymbol{A}-\boldsymbol{D}$, Eps8 KD does not affect the total number of synapses containing surface GluA1, but it induces a decrease in the number of synapses on spines with a concomitant increase in the number of shaft synapses ( $\boldsymbol{A}$, arrowheads) containing surface GluA1. Scale bar, $5 \mu \mathrm{m}$. $\boldsymbol{E}$, AMPA receptor-mediated mEPSCs were recorded from cultured neurons. Eps8 knockdown does not affect the frequency $(\boldsymbol{F})$ or the amplitude $(\boldsymbol{G})$ of $\mathrm{mEPSC}$. Representative $10 \mathrm{~s}$ traces of $\mathrm{mEPSC}$ illustrating similar frequency and amplitude in scrambled and Eps8 shRNA-expressing cells. ${ }^{* *} p<0.01$. ${ }^{* * *} p<0.001$.

that LTP increases both the number and size of dendritic spines (Segal, 2005; Alvarez and Sabatini, 2007; Bosch and Hayashi, 2011). Actin cytoskeleton dynamics are required for activitymediated spine morphological changes (Bramham, 2008). We therefore decided to examine the contribution of Eps8 to LTPmediated spine plasticity. To address this question, we used a cLTP protocol, which consists of the activation of NMDA receptors with glycine in the absence of $\mathrm{Mg}^{2+}$. As previously reported (Fortin et al., 2010; Keith et al., 2012), this protocol induces spine formation and enlargement. Indeed, in control scrambled shRNA-expressing neurons, cLTP significantly increases both the number and the size of dendritic spines (Fig. $6 A-C$ ). In contrast, Eps8 shRNAs-expressing neurons do not respond to the cLTP stimulus, as spine density and size remain unchanged (Fig. 6AC). Thus, Eps8 is required for LTP-dependent structural plasticity of dendritic spines.

To examine the role of Eps8 on LTP-mediated functional plasticity, we recorded AMPA receptor-mediated mEPSCs (Fig.
D

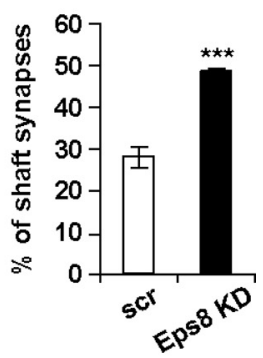

G

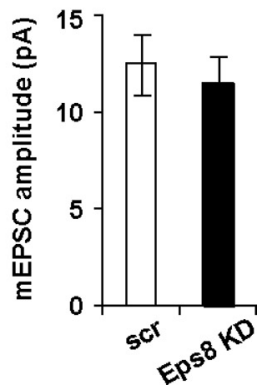

$6 D)$. We found that cLTP increases both mEPSC frequency $(180 \%)$ and amplitude $(48 \%)$ in scrambled shRNA-expressing cells (Fig. $6 E, F$ ), as previously shown in cultured hippocampal neurons ( $\mathrm{Oh}$ and Derkach, 2005; Fortin et al., 2010; Keith et al., 2012). In contrast, Eps8 silencing completely blocks the effect of cLTP on mEPSC frequency. However, the amplitude was significantly increased by $38 \%$ after cLTP (Fig. 6E,F), as observed in scrambled shRNA-expressing neurons. These results indicate that Eps8 is required for LTP-mediated synapse formation but not for LTP-induced synaptic strengthening.

\section{Discussion}

Long-term changes in synaptic activity have profound effects on the formation and morphology of dendritic spines and synaptic strength (Alvarez and Sabatini, 2007; Kasai et al., 2010; Bosch and Hayashi, 2011). Indeed, LTP increases spine density and spine growth through modifications of the actin cytoskeleton (Cingolani and Goda, 2008; Hotulainen and Hoogenraad, 2010; Bosch and Hayashi, 2011). However, little is known about the molecular mechanisms by which neuronal activity regulates the actin cytoskeleton during spine plasticity. Here, we demonstrate that the actin-capping protein Eps8 modulates spine morphogenesis and is required for LTP-mediated spine formation and some aspects of synaptic potentiation.

Eps8 is a multifunctional protein that induces actin remodeling through its bundling and capping activities or through Racl activation (Disanza et al., 2004; RoffersAgarwal et al., 2005; Disanza et al., 2006; Menna et al., 2009; Hertzog et al., 2010). Interestingly, Eps8 can either induce or inhibit filopodium formation (Disanza et al., 2006; Menna et al., 2009). These activities are highly dependent on the cellular context and are regulated by interactions with its partners IRSp53 and Abi1/2 (Vaggi et al., 2011). In HeLa cells, Eps8 promotes filopodium formation through the interaction with IRSp53 via its actin bundling activity (Disanza et al., 2006; Vaggi et al., 2011). In neurons, in contrast, Eps8 inhibits filopodium formation through its capping activity (Menna et al., 2009). Consistent with this finding, we show that Eps8 inhibits filopodium formation in dendrites (Figs. $1 E$ and $2 F$ ). These results suggest that the bundling activity of Eps8 is not required for the formation of filopodia in neurons.

In neurons, Eps8 is not required for Rac1 activation, as Rac activity is not affected in Eps8 KO mice (Menna et al., 2009). In addition, Racl inhibition does not mimic the effect of Eps8 loss of function in the formation of axonal filopodia in neurons (Kozma et al., 1997; Menna et al., 2009). Consistent with these findings, we found that the Rac1 inhibitor (NSC23766) does not block Eps8 function on spines (data not shown). In addition, we found 
that a mutant Eps8 (Eps8TM), defective in actin capping but not in Racl activation, mimics the loss of function of Eps8. Together, these results suggest that Eps8 regulates spine morphogenesis through a pathway independent of Rac1.

Barbed end actin-capping proteins are of major importance in the regulation of actin dynamics by inhibiting actin filament elongation. Defects in capping proteins result in the formation of long actin filaments and promote excessive filopodium formation (Mejillano et al., 2004; Menna et al., 2009; Fan et al., 2011). Indeed, Eps8 loss of function increases the amount of filamentous actin (Vaggi et al., 2011) and axonal filopodia in neurons (Menna et al., 2009). In agreement with these findings, we show that Eps8 loss of function increases, whereas gain of function decreases, the number of dendritic filopodia in hippocampal neurons. Gainand loss-of-function studies demonstrate that Eps8 regulates the number of dendritic spines. Moreover, Eps8 KD results in bigger spines with irregular shape and filopodial protrusions emerging from spine heads. The structural effects of Eps8 $\mathrm{KD}$ on dendritic filopodia and spines are similar to those observed when CP, an actin-capping protein, is silenced (Fan et al., 2011). These findings are consistent with the view that Eps8 acts as a capping protein to regulate filopodium and spine formation.

Several experiments demonstrate that Eps8 regulates spine morphogenesis through its actin-capping activity. First, a mutant Eps8 carrying three point mutations (V729A, T731A, and W732A), which specifically abolish its capping activity (Menna et al., 2009), exhibits defects in spine morphogenesis mimicking the loss of function of Eps8. Second, Eps8 loss of function increases F-actin accumulation at dendritic spines as expected for a capping protein. Third, free-barbed end assays and FRAP experiments demonstrate that Eps8 KD increases the level of uncapped barbed ends available for actin polymerization and induces fast actin turnover within dendritic spines. Together, our results strongly suggest that Eps8 regulates spine formation and morphology through its actin-capping activity.

Although most excitatory synapses are formed on dendritic spines, some synapses are present on the dendritic shaft. Shaft synapses are mainly present in young neurons and therefore might represent spine precursors during early stages of synaptogenesis (Boyer et al., 1998; Fiala et al., 1998; Reilly et al., 2011). However, very little is known about the mechanisms that regulate the formation of spine versus shaft synapses. For example, silencing of neurobeachin, a protein that regulates membrane traffick-

A

D
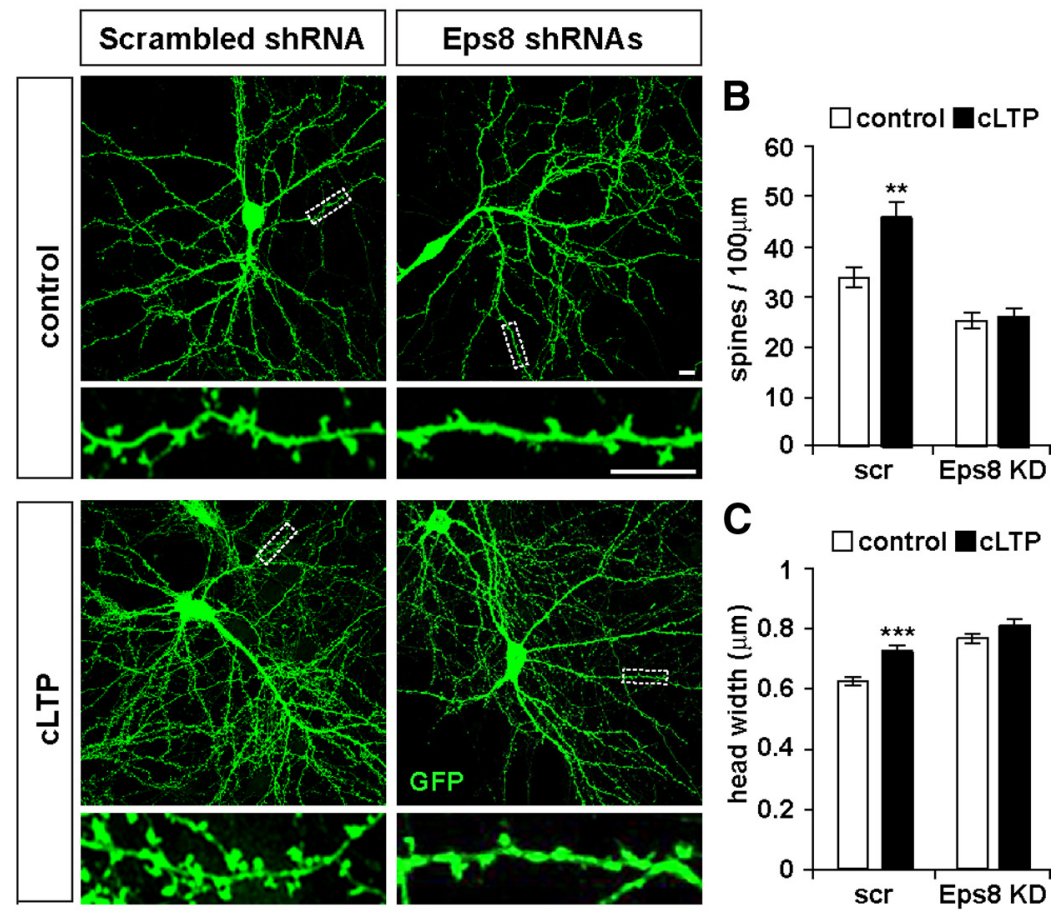

Scrambled shRNA control

Scrambled shRNA cLTP

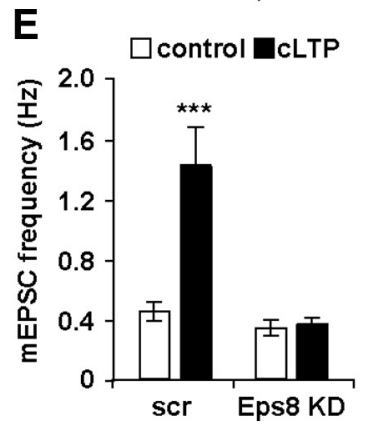

Eps8 shRNAs control

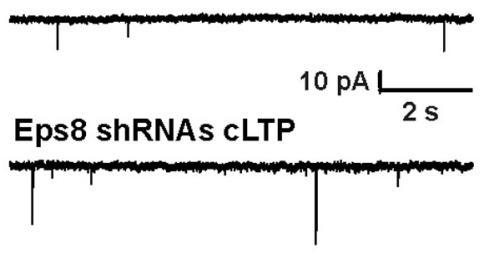

$\mathbf{F}$

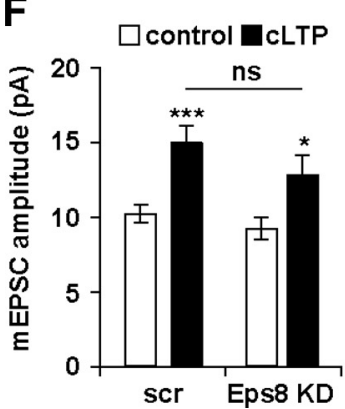

Figure 6. Eps8 is required for activity-dependent spine formation. CLTP was induced in hippocampal neurons expressing scrambled shRNA or shRNAs against Eps8 (A). Scale bars, $10 \mu \mathrm{m}$. In scrambled shRNA-expressing cells, LLTP increases spine density $(\boldsymbol{B})$ and size ( $\boldsymbol{C}$. In neurons expressing Eps8 shRNAs, LLT does not affect the number or the size of dendritic spines ( $\boldsymbol{B}, \boldsymbol{C}$ ). D, AMPA receptor-mediated $\mathrm{mEPSCS}$ were recorded from cultured neurons in control condition or after CLTP induction. Representative $10 \mathrm{~s}$ traces of mEPSCs are shown (D). Scrambled shRNA-expressing cells show a significant increase in both mEPSC frequency and amplitude $(\boldsymbol{E}, \boldsymbol{F})$. In contrast, CLTP in Eps8 $\mathrm{KD}$ has no effect in mEPSC frequency $(\boldsymbol{E})$ but induces a significant increase in the amplitude of mEPSCs $(\boldsymbol{F}) .{ }^{*} p<0.05 .{ }^{* *} p<0.01 .{ }^{* * *} p<0.001$. ns, Not significant.

ing, decreases spine density with a concomitant increase in the number of excitatory shaft synapses (Niesmann et al., 2011). Similar effects are observed in the gain of function of Rap2, a small GTPase (Fu et al., 2007). Here, we showed that Eps8 promotes the formation of excitatory synapses on spines with a concomitant decrease in the number of excitatory shaft synapses. In contrast, Eps8 KD induces the opposite effect. Several studies have shown that increased shaft synapse density does not affect spontaneous 
synaptic currents, when the total number of synapses is unchanged (Aoto et al., 2007; Fu et al., 2007; Ivenshitz and Segal, 2010; Niesmann et al., 2011). Indeed, we found that Eps8 KD does not affect the frequency or the amplitude of mEPSCs in hippocampal neurons under basal conditions. Consistent with our findings, spontaneous synaptic currents are unaffected in cerebellar granule neurons from Eps8 $\mathrm{KO}$ mice (Offenhauser et al., 2006). In summary, our findings demonstrate that Eps8 regulates the balance between spine and shaft excitatory synapses without affecting basal synaptic transmission.

Activity-dependent changes in spine plasticity are highly dependent on local changes in the actin cytoskeleton (Fukazawa et al., 2003; Okamoto et al., 2004; Honkura et al., 2008; Gu et al., 2010). Indeed, neuronal activity regulates actin turnover within dendritic spines (Star et al., 2002; Okamoto et al., 2004; Honkura et al., 2008). Barbed end capping proteins regulate actin turnover by inhibiting filament elongation, therefore maintaining the equilibrium between monomeric (G-actin) and filamentous actin (F-actin). Although the role of capping proteins in spine morphogenesis has been documented (Fan et al., 2011; Gao et al., 2011), their function in activity-mediated changes has not been reported. Here, we demonstrate that Eps8 silencing suppresses the effect of cLTP on spine morphogenesis, demonstrating that Eps8 is required for activity-mediated spine structural plasticity.

Eps8 is required for some aspects of LTP-mediated synaptic plasticity. We found that Eps8 KD impairs LTP-mediated increase in the frequency, but not in the amplitude, of AMPARmediated mEPSCs, suggesting that, in the absence Eps8, an LTP stimulus can still increase the density and/or function of AMPARs at the synapse. Interestingly, previous studies have shown that actin polymerization is crucial for the maintenance, but not the induction, of LTP (Krucker et al., 2000; Fukazawa et al., 2003; Ramachandran and Frey, 2009). Moreover, it has been suggested that different pools of actin are present at spines to differentially regulate different processes (Star et al., 2002; Okamoto et al., 2004; Honkura et al., 2008). Based on these findings, we propose that Eps8, through its capping activity, regulates a pool of actin required for the late stages of LTP without affecting LTPmediated increases in synaptic strength.

\section{References}

Alvarez VA, Sabatini BL (2007) Anatomical and physiological plasticity of dendritic spines. Annu Rev Neurosci 30:79-97. CrossRef Medline

Aoto J, Ting P, Maghsoodi B, Xu N, Henkemeyer M, Chen L (2007) Postsynaptic ephrinB3 promotes shaft glutamatergic synapse formation. J Neurosci 27:7508-7519. CrossRef Medline

Bosch M, Hayashi Y (2012) Structural plasticity of dendritic spines. Curr Opin Neurobiol 22:383-388. CrossRef Medline

Bourne JN, Harris KM (2008) Balancing structure and function at hippocampal dendritic spines. Annu Rev Neurosci 31:47-67. CrossRef Medline

Boyer C, Schikorski T, Stevens CF (1998) Comparison of hippocampal dendritic spines in culture and in brain. J Neurosci 18:5294-5300. Medline

Bramham CR (2008) Local protein synthesis, actin dynamics, and LTP consolidation. Curr Opin Neurobiol 18:524-531. CrossRef Medline

Carlisle HJ, Kennedy MB (2005) Spine architecture and synaptic plasticity. Trends Neurosci 28:182-187. CrossRef Medline

Ciani L, Boyle KA, Dickins E, Sahores M, Anane D, Lopes DM, Gibb AJ, Salinas PC (2011) Wnt7a signaling promotes dendritic spine growth and synaptic strength through $\mathrm{Ca}(2) / \mathrm{calmodulin}$-dependent protein kinase II. Proc Natl Acad Sci U S A 108:10732-10737. CrossRef Medline

Cingolani LA, Goda Y (2008) Actin in action: the interplay between the actin cytoskeleton and synaptic efficacy. Nat Rev Neurosci 9:344-356. CrossRef Medline

Disanza A, Carlier MF, Stradal TE, Didry D, Frittoli E, Confalonieri S, Croce A, Wehland J, Di Fiore PP, Scita G (2004) Eps8 controls actin-based motility by capping the barbed ends of actin filaments. Nat Cell Biol 6:1180-1188. CrossRef Medline

Disanza A, Mantoani S, Hertzog M, Gerboth S, Frittoli E, Steffen A, Berhoerster K, Kreienkamp HJ, Milanesi F, Di Fiore PP, Ciliberto A, Stradal TE, Scita G (2006) Regulation of cell shape by Cdc42 is mediated by the synergic actin-bundling activity of the Eps8-IRSp53 complex. Nat Cell Biol 8:1337-1347. CrossRef Medline

Dotti CG, Sullivan CA, Banker GA (1988) The establishment of polarity by hippocampal neurons in culture. J Neurosci 8:1454-1468. Medline

Dudek H, Ghosh A, Greenberg ME (2001) Calcium phosphate transfection of DNA into neurons in primary culture. Curr Protoc Neurosci Chapter 3:Unit 3.11. CrossRef Medline

Fan Y, Tang X, Vitriol E, Chen G, Zheng JQ (2011) Actin capping protein is required for dendritic spine development and synapse formation. J Neurosci 31:10228-10233. CrossRef Medline

Fiala JC, Feinberg M, Popov V, Harris KM (1998) Synaptogenesis via dendritic filopodia in developing hippocampal area CAl. J Neurosci 18: 8900-8911. Medline

Fortin DA, Davare MA, Srivastava T, Brady JD, Nygaard S, Derkach VA, Soderling TR (2010) Long-term potentiation-dependent spine enlargement requires synaptic $\mathrm{Ca}^{2+}$-permeable AMPA receptors recruited by CaM-kinase I. J Neurosci 30:11565-11575. CrossRef Medline

Fu Z, Lee SH, Simonetta A, Hansen J, Sheng M, Pak DT (2007) Differential roles of Rap1 and Rap2 small GTPases in neurite retraction and synapse elimination in hippocampal spiny neurons. J Neurochem 100:118-131. CrossRef Medline

Fukazawa Y, Saitoh Y, Ozawa F, Ohta Y, Mizuno K, Inokuchi K (2003) Hippocampal LTP is accompanied by enhanced F-actin content within the dendritic spine that is essential for late LTP maintenance in vivo. Neuron 38:447-460. CrossRef Medline

Gao C, Frausto SF, Guedea AL, Tronson NC, Jovasevic V, Leaderbrand K, Corcoran KA, Guzmán YF, Swanson GT, Radulovic J (2011) IQGAP1 regulates NR2A signaling, spine density, and cognitive processes. J Neurosci 31:8533-8542. CrossRef Medline

Gu J, Lee CW, Fan Y, Komlos D, Tang X, Sun C, Yu K, Hartzell HC, Chen G, Bamburg JR, Zheng JQ (2010) ADF/cofilin-mediated actin dynamics regulate AMPA receptor trafficking during synaptic plasticity. Nat Neurosci 13:1208-1215. CrossRef Medline

Han K, Kim E (2008) Synaptic adhesion molecules and PSD-95. Prog Neurobiol 84:263-283. CrossRef Medline

Hertzog M, Milanesi F, Hazelwood L, Disanza A, Liu H, Perlade E, Malabarba MG, Pasqualato S, Maiolica A, Confalonieri S, Le Clainche C, Offenhauser N, Block J, Rottner K, Di Fiore PP, Carlier MF, Volkmann N, Hanein D, Scita G (2010) Molecular basis for the dual function of Eps8 on actin dynamics: bundling and capping. PLoS Biol 8:e1000387. CrossRef Medline

Honkura N, Matsuzaki M, Noguchi J, Ellis-Davies GC, Kasai H (2008) The subspine organization of actin fibers regulates the structure and plasticity of dendritic spines. Neuron 57:719-729. CrossRef Medline

Hotulainen P, Hoogenraad CC (2010) Actin in dendritic spines: connecting dynamics to function. J Cell Biol 189:619-629. CrossRef Medline

Innocenti M, Tenca P, Frittoli E, Faretta M, Tocchetti A, Di Fiore PP, Scita G (2002) Mechanisms through which Sos-1 coordinates the activation of Ras and Rac. J Cell Biol 156:125-136. CrossRef Medline

Ivenshitz M, Segal M (2010) Neuronal density determines network connectivity and spontaneous activity in cultured hippocampus. J Neurophysiol 104:1052-1060. CrossRef Medline

Kasai H, Fukuda M, Watanabe S, Hayashi-Takagi A, Noguchi J (2010) Structural dynamics of dendritic spines in memory and cognition. Trends Neurosci 33:121-129. CrossRef Medline

Keith DJ, Sanderson JL, Gibson ES, Woolfrey KM, Robertson HR, Olszewski K, Kang R, El-Husseini A, Dell'acqua ML (2012) Palmitoylation of a-kinase anchoring protein 79/150 regulates dendritic endosomal targeting and synaptic plasticity mechanisms. J Neurosci 32:7119-7136. CrossRef Medline

Koskinen M, Bertling E, Hotulainen P (2012) Methods to measure actin treadmilling rate in dendritic spines. Methods Enzymol 505:47-58. CrossRef Medline

Kozma R, Sarner S, Ahmed S, Lim L (1997) Rho family GTPases and neuronal growth cone remodelling: relationship between increased complexity induced by $\mathrm{Cdc} 42 \mathrm{Hs}$, Racl, and acetylcholine and collapse induced by RhoA and lysophosphatidic acid. Mol Cell Biol 17:1201-1211. Medline 
Krucker T, Siggins GR, Halpain S (2000) Dynamic actin filaments are required for stable long-term potentiation (LTP) in area CA1 of the hippocampus. Proc Natl Acad Sci U S A 97:6856-6861. CrossRef Medline

Marsick BM, Letourneau PC (2011) Labeling F-actin barbed ends with rhodamine-actin in permeabilized neuronal growth cones. J Vis Exp 17:49. pii: 2409. CrossRef Medline

Matsuzaki M, Ellis-Davies GC, Nemoto T, Miyashita Y, Iino M, Kasai H (2001) Dendritic spine geometry is critical for AMPA receptor expression in hippocampal CA1 pyramidal neurons. Nat Neurosci 4:1086-1092. CrossRef Medline

McMahon SA, Díaz E (2011) Mechanisms of excitatory synapse maturation by trans-synaptic organizing complexes. Curr Opin Neurobiol 21:221-227. CrossRef Medline

Mejillano MR, Kojima S, Applewhite DA, Gertler FB, Svitkina TM, Borisy GG (2004) Lamellipodial versus filopodial mode of the actin nanomachinery: pivotal role of the filament barbed end. Cell 118:363-373. CrossRef Medline

Menna E, Disanza A, Cagnoli C, Schenk U, Gelsomino G, Frittoli E, Hertzog M, Offenhauser N, Sawallisch C, Kreienkamp HJ, Gertler FB, Di Fiore PP, Scita G, Matteoli M (2009) Eps8 regulates axonal filopodia in hippocampal neurons in response to brain-derived neurotrophic factor (BDNF). PLoS Biol 7:e1000138. CrossRef Medline

Niesmann K, Breuer D, Brockhaus J, Born G, Wolff I, Reissner C, Kilimann MW, Rohlmann A, Missler M (2011) Dendritic spine formation and synaptic function require neurobeachin. Nat Commun 2:557. CrossRef Medline

Offenhäuser N, Borgonovo A, Disanza A, Romano P, Ponzanelli I, Iannolo G, Di Fiore PP, Scita G (2004) The eps8 family of proteins links growth factor stimulation to actin reorganization generating functional redundancy in the Ras/Rac pathway. Mol Biol Cell 15:91-98. CrossRef Medline

Offenhäuser N, Castelletti D, Mapelli L, Soppo BE, Regondi MC, Rossi P, D’Angelo E, Frassoni C, Amadeo A, Tocchetti A, Pozzi B, Disanza A, Guarnieri D, Betsholtz C, Scita G, Heberlein U, Di Fiore PP (2006) Increased ethanol resistance and consumption in Eps8 knockout mice correlates with altered actin dynamics. Cell 127:213-226. CrossRef Medline

Oh MC, Derkach VA (2005) Dominant role of the GluR2 subunit in regulation of AMPA receptors by CaMKII. Nat Neurosci 8:853-854. CrossRef Medline

Okamoto K, Nagai T, Miyawaki A, Hayashi Y (2004) Rapid and persistent modulation of actin dynamics regulates postsynaptic reorganization underlying bidirectional plasticity. Nat Neurosci 7:1104-1112. CrossRef Medline

Proepper C, Johannsen S, Liebau S, Dahl J, Vaida B, Bockmann J, Kreutz MR, Gundelfinger ED, Boeckers TM (2007) Abelson interacting protein 1
(Abi-1) is essential for dendrite morphogenesis and synapse formation. EMBO J 26:1397-1409. CrossRef Medline

Ramachandran B, Frey JU (2009) Interfering with the actin network and its effect on long-term potentiation and synaptic tagging in hippocampal CA1 neurons in slices in vitro. J Neurosci 29:12167-12173. CrossRef Medline

Reilly JE, Hanson HH, Phillips GR (2011) Persistence of excitatory shaft synapses adjacent to newly emerged dendritic protrusions. Mol Cell Neurosci 48:129-136. CrossRef Medline

Roffers-Agarwal J, Xanthos JB, Miller JR (2005) Regulation of actin cytoskeleton architecture by Eps8 and Abil. BMC Cell Biol 6:36. CrossRef Medline

Sahores M, Gibb A, Salinas PC (2010) Frizzled-5, a receptor for the synaptic organizer Wnt7a, regulates activity-mediated synaptogenesis. Development 137:2215-2225. CrossRef Medline

Schubert V, Dotti CG (2007) Transmitting on actin: synaptic control of dendritic architecture. J Cell Sci 120:205-212. CrossRef Medline

Scita G, Nordstrom J, Carbone R, Tenca P, Giardina G, Gutkind S, Bjarnegård M, Betsholtz C, Di Fiore PP (1999) EPS8 and E3B1 transduce signals from Ras to Rac. Nature 401:290-293. CrossRef Medline

Scita G, Tenca P, Areces LB, Tocchetti A, Frittoli E, Giardina G, Ponzanelli I, Sini P, Innocenti M, Di Fiore PP (2001) An effector region in Eps8 is responsible for the activation of the Rac-specific GEF activity of Sos-1 and for the proper localization of the Rac-based actin-polymerizing machine. J Cell Biol 154:1031-1044. CrossRef Medline

Segal M (2005) Dendritic spines and long-term plasticity. Nat Rev Neurosci 6:277-284. CrossRef Medline

Segal M (2010) Dendritic spines, synaptic plasticity and neuronal survival: activity shapes dendritic spines to enhance neuronal viability. Eur J Neurosci 31:2178-2184. CrossRef Medline

Sekerková G, Diño MR, Ilijic E, Russo M, Zheng L, Bartles JR, Mugnaini E (2007) Postsynaptic enrichment of Eps8 at dendritic shaft synapses of unipolar brush cells in rat cerebellum. Neuroscience 145:116-129. CrossRef Medline

Star EN, Kwiatkowski DJ, Murthy VN (2002) Rapid turnover of actin in dendritic spines and its regulation by activity. Nat Neurosci 5:239-246. CrossRef Medline

Tada T, Sheng M (2006) Molecular mechanisms of dendritic spine morphogenesis. Curr Opin Neurobiol 16:95-101. CrossRef Medline

Vaggi F, Disanza A, Milanesi F, Di Fiore PP, Menna E, Matteoli M, Gov NS, Scita G, Ciliberto A (2011) The Eps8/IRSp53/VASP network differentially controls actin capping and bundling in filopodia formation. PLoS Comput Biol 7:e1002088. CrossRef Medline 